\title{
A NOTE ON STRICTLY CYCLIC WEIGHTED SHIFTS
}

\author{
HECTOR N. SALAS
}

ABSTRACT. We answer negatively Shields' Question 15 in [1].

The object of this note is to answer negatively one of the many questions raised by Allen L. Shields in his article [1]. Question 15 of [1] is: If $T$ is a unilateral weighted shift with weights $w_{n}$ decreasing to 1 and if $\sum_{n=0}^{\infty} \beta(n)^{-2}<\infty$ where $\beta(n)=w_{0} w_{1} \cdots w_{n-1}$, must $T$ be strictly cyclic? If $\left\{w_{n}\right\}$ decreases to 0 , must $T$ be strictly cyclic? Strongly strictly cyclic?

By Proposition 32 of $[1$, p. 96], to answer the question negatively it will suffice to show that the supremum

$$
\operatorname{Sup}_{n} \sum_{k=0}^{n}\left(\frac{\beta(n)}{\beta(k) \beta(n-k)}\right)^{2}
$$

is infinite.

For the first part of the question, we let $\left\{a_{j}\right\}$ be any sequence decreasing to 1 and we choose an increasing sequence of positive integers so that

$$
\left[a_{1}^{n_{1}} a_{2}^{n_{2}-n_{1}} \cdots a_{j}^{n_{j}-n_{j-1}}\right]^{-2}\left(\frac{a_{j+1}^{2}}{a_{j+1}^{2}-1}\right)<2^{-j}
$$

and

$$
\left(1+n_{j}\right)\left(\frac{a_{j}}{a_{1}}\right)^{2 n_{j-1}}>j
$$

Such a choice is clearly possible. We set $w_{n}=a_{1}$, if $0<n<n_{1}$, and we set $w_{n}=a_{j}$, if $n_{j-1} \leqslant n<n_{j}$. In order to verify that our selection works we use (2) to show that $\sum_{n>0} \beta(n)^{-2}<a_{1}^{2} /\left(a_{1}^{2}-1\right)+1$ and (3) to show that the supremum (1) is infinite.

Consider (2) first. We have

$$
\sum_{n=0}^{\infty} \frac{1}{\beta(n)^{2}}=\sum_{n=0}^{n_{1}} \frac{1}{\beta(n)^{2}}+\sum_{j=2}^{\infty}\left(\sum_{n_{j-1}<n<n_{j}} \frac{1}{\beta(n)^{2}}\right)
$$

where $\beta(n)$ is given by the equations $\beta(n)=a_{1}^{n}$ if $n<n_{1}$, and $\beta(n)=$ $a_{1}^{n_{1}} a_{2}^{n_{2}-n_{1}} \cdots a_{j}^{n-n_{j-1}}$ if $n_{j-1}<n \leqslant n_{j}$.

Received by the editors March 12, 1981.

1980 Mathematics Subject Classification. Primary 47B37. 
Therefore, $\sum_{n=0}^{n_{1}} \beta(n)^{-2}<\sum_{n=0}^{\infty} a_{1}^{-2 n}=a_{1}^{2} /\left(a_{1}^{2}-1\right)$, while

$$
\begin{aligned}
\sum_{n_{j-1}<n<n_{j}} \frac{1}{\beta(n)^{2}} & =\left[a_{1}^{n_{1}} a^{n_{2}-n_{1}} \cdots a_{j-1}^{n_{j-1}-n_{j-2}}\right]^{-2}\left(\sum_{n_{j-1}<n<n_{j}} \frac{1}{a_{j}^{2\left(n-n_{j-1}\right)}}\right) \\
& <\left[a_{1}^{n_{1}} \cdots a_{j-1}^{n_{j-1}-n_{j-2}}\right]^{-2}\left(\frac{a_{j}^{2}}{a_{j}^{2}-1}\right)<2^{-j+1} .
\end{aligned}
$$

This with (4) shows that $\sum_{n=0}^{\infty} \beta(n)^{-2}<a_{1}^{2} /\left(a_{1}^{2}-1\right)+1$.

To show that (1) is violated we show that a lower bound for

$$
\sum_{k=0}^{n_{j}}\left(\frac{\beta\left(n_{j}\right)}{\beta(k) \beta\left(n_{j}-k\right)}\right)^{2}
$$

is $j$. Consider

$$
\frac{\beta\left(n_{j}\right)}{\beta(k) \beta\left(n_{j}-k\right)}=\frac{w_{k} \cdots w_{n_{j}-1}}{w_{0} \cdots w_{n_{j}-(k+1)}} ;
$$

in both top and bottom there are no more than $n_{j-1}$ weights different from $a_{j}$. Thus, we can say that $\beta\left(n_{j}\right) / \beta(k) \beta\left(n_{j}-k\right)>\left(a_{j} / a_{1}\right)^{n_{j-1}}$, which together with (3) gives (5).

Note that it is actually possible to choose the sequence $\left\{w_{n}\right\}$ strictly decreasing if desired. One might ask if it is possible to choose the weight sequence to be convex.

The second part of the question is answered with a similar construction. Simply choose $\left\{a_{j}\right\}$ decreasing to zero and choose $\left\{n_{j}\right\}$ so that $(3)$ is satisfied. If $\left\{w_{n}\right\}$ is defined as before, then the supremum (1) is infinite, and $T$ is not strictly cyclic.

\section{REFERENCES}

1. A. L. Shields, Weighted shift operators and analytic function theory, Math. Surveys No. 13, Amer. Math. Soc., Providence, R.I., 1974.

Department of Mathematics, University of Iowa, Iowa City, Iowa 52242 